\title{
Length scales for the Navier-Stokes equations on a rotating sphere
}

\author{
Yuliya N. Kyrychko ${ }^{*}, 1$ and Michele V. Bartuccelli \\ Department of Mathematics and Statistics, University of Surrey, \\ Guildford, Surrey GU2 7XH, UK
}

\begin{abstract}
In this letter we obtain the dissipative length scale for the Navier-Stokes equations on a two-dimensional rotating sphere $S^{2}$. This system is a fundamental model of the large scale atmospheric dynamics. Using the equations of motion in their vorticity form, we construct the ladder inequalities from which a set of time-averaged length scales is obtained.
\end{abstract}

Key words: Length scales, Navier-Stokes equations, Rotating sphere.

PACS: $47.27 .-\mathrm{i}$

\section{Introduction}

The Navier-Stokes equations is a fundamental model which naturally arises in different aspects of meteorology. This system on a rotating sphere is a simple model of the large scale atmospheric dynamics and is often used in

* Corresponding author.

Email address: y.kyrychko@eim.surrey .ac.uk (Yuliya N. Kyrychko).

1 Tel.: +44 1483 682642; fax: +44 1483686071 .

Preprint submitted to Elsevier Science

24 March 2004 
meteorological modelling in the vorticity formulation. In particular, it has been used to study the two-dimensional turbulence on a rotating sphere in the context of atmospheric circulation on large planets [1].

In recent years there has been a growing interest in the study of the NavierStokes system on manifolds. The best estimate of the attractor dimension for the Navier-Stokes equations was obtained by Ilyin [2]. In [3] a simple proof of the explicit estimates of the attractor dimension for the Navier-Stokes system on a rotating sphere in terms of the vorticity equation has been performed. The problem of upper bounds for the Hausdorff and fractal dimensions of the global attractor for the Navier-Stokes equations on a two-dimensional compact manifold was addressed in [2]. In particular, in the case of the unit sphere the author gives the explicit values of the constants involved. Cao, Rammaha and Titi [4] have proved the Gevrey regularity for the Navier-Stokes equation on the two-dimensional sphere $S^{2}$ and found an upper bound for the number of asymptotic degrees of freedom for this system. Global existence of strong solutions on the sphere has been studied in [5-7].

The aim of this letter is to find dissipative length scales estimates for the solutions of the Navier-Stokes system on a rotating sphere using the vorticity equation and the best to date interpolation inequalities on the sphere. The length scales serve for better understanding the spatio-temporal dynamics of the solutions of dissipative PDEs, providing information about the smallest features of the flow. In order to find the estimates for the length scales we use the so-called ladder method which has been applied to the wide range of partial differential equations such as the complex Ginzburg-Landau equation [8] and the Navier-Stokes equations on a flat domains with periodic boundary conditions $[9]$. 
Consider the Navier-Stokes equations of an incompressible fluid on a twodimensional rotating sphere $S^{2}$ in the following form [2,3]:

$$
\begin{gathered}
\frac{\partial u}{\partial t}+\nu \operatorname{rot}_{\operatorname{rot}_{n}} u+\nabla\left(\frac{u^{2}}{2}\right)-u \times \operatorname{rot}_{n} u+\ell \vec{n} \times u+\nabla p=f, \\
\operatorname{div} u=0,
\end{gathered}
$$

where $\nu>0$ is the viscosity constant, $u$ is a velocity vector in the tangent bundle $T S^{2}, p$ is a scalar function for pressure, $f$ is a baroclinic forcing term, " $\times$ " denotes the standard vector product, $R$ is the radius of the sphere, $\ell=$ $2 \omega \sin \phi$ is the Coriolis parameter, $\omega$ is an angular velocity, $|\phi| \leq \frac{\pi}{2}$ is a latitude, $\theta$ is a longitude $(0 \leq \theta \leq 2 \pi), \vec{n}$ is the outward unit normal vector to $S^{2}$. By definition, the operator $\operatorname{rot}_{n} u$ is [2]

$$
\operatorname{rot}_{n} u=-\vec{n} \operatorname{div}(\vec{n} \times u)=(\operatorname{rot} u \cdot \vec{n}) \vec{n},
$$

and

$$
\operatorname{rot} h=-\vec{n} \times \nabla h,
$$

where $u$ is a vector field and $h$ is a scalar function. It is easy to see that

$$
\operatorname{rot}_{n} \operatorname{rot} h=-\vec{n}(\Delta h)
$$

The inner products for the $L^{2}$-spaces of scalar functions denoted by $L^{2}\left(S^{2}\right)$ and the $L^{2}$-spaces of the tangent vector fields on $S^{2}$ denoted by $L^{2}\left(T S^{2}\right)$ are given by [4]:

$$
\begin{gathered}
(u, v)_{L^{2}\left(S^{2}\right)}=\int_{S^{2}} u v d S, \quad \text { for } u, v \in L^{2}\left(S^{2}\right) \\
(u, v)_{L^{2}\left(T S^{2}\right)}=\int_{S^{2}} u \cdot v d S, \quad \text { for } \quad u, v \in L^{2}\left(T S^{2}\right) .
\end{gathered}
$$

Whenever we integrate by parts we shall use the following formulas [4]

$$
(\nabla h, v)=(\nabla h, v)_{L^{2}\left(T S^{2}\right)}=-(h, \operatorname{div} v)_{L^{2}\left(S^{2}\right)},
$$




$$
(\operatorname{rot} u, v)=(\operatorname{rot} u, v)_{L^{2}\left(T S^{2}\right)}=\left(u, \operatorname{rot}_{n} v\right)_{L^{2}\left(T S^{2}\right)}, \quad \text { for } \quad u, v \in L^{2}\left(T S^{2}\right)
$$

The ladder method involves replacing the original PDE by a hierarchy of ordinary differential inequalities. These inequalities are then used to control the norms of all spatial derivatives of the solution of the equation, under the assumption that one can obtain an upper bound on the "bottom" rung of the ladder. For our purposes it will be more convenient to work with the scalar equation. Therefore, introducing $\psi$ as a stream function for $u: u=\vec{n} \times \nabla \psi$, $\psi=\Delta^{-1}\left(\vec{n} \cdot \operatorname{rot}_{n} u\right)$, substituting this in the equation (1), and applying the operator $\operatorname{rot}_{n}$ we obtain a vorticity equation [3]:

$$
\partial_{t} \Delta \psi+J(\psi, \Delta \psi+l)-\nu \Delta^{2} \psi=\vec{n} \cdot \operatorname{rot}_{n} f,
$$

where we followed the notations from [2,3]: $J(a, b)=\vec{n} \times \nabla a \cdot \nabla b$, and in particular $J(\psi, \Delta \psi+l)=u \cdot \nabla(\Delta \psi+l)$. The function $J(a, b)$ has the property [2]:

$$
\int_{S^{2}} J(a, b) d s=0,
$$

and it can also be proved that

$$
\int_{S^{2}} J(\psi, \varphi) \varphi d s=0, \int_{S^{2}} J(\psi, l) \Delta \psi d s=0 .
$$

Finally, let $\varphi=\Delta \psi$ and introduce spaces $H=L_{2}\left(S^{2}\right) \cup\left\{\varphi: \int \varphi d s=0\right\}$ and $H_{1}=H^{1}\left(S^{2}\right) \cup H$. Hence, the equation (5) takes the form

$$
\partial_{t} \varphi+J\left(\Delta^{-1} \varphi, \varphi+l\right)-\nu \Delta \varphi=\vec{n} \cdot \operatorname{rot}_{n} f, \varphi(0)=\varphi_{0} .
$$

In order to calculate the length scales we define the following time-dependent functionals $H_{N}, \mathcal{F}_{N}$ and $F_{N}$ as

$$
H_{N}:=\left\|\nabla^{N} \varphi\right\|_{2}^{2}=\int_{S^{2}}\left|\nabla^{N} \varphi\right|^{2} d S
$$




$$
\mathcal{F}_{N}:=\int_{S^{2}}\left|\nabla^{N}\left(\vec{n} \cdot \operatorname{rot}_{n} f\right)\right|^{2} d S
$$

and we also introduce a natural "time" $\tau=R^{2} \nu^{-1}$ which is based on the radius of the sphere $R$ and the viscosity $\nu$. Then

$$
F_{N}=H_{N}+\tau^{2} \mathcal{F}_{N}
$$

We assume a forcing to be time-independent and such that the forcing function $f(x)$ has a cut-off in its spectrum such that it has the smallest length scale

$$
\lambda_{f}=\sup _{N}\left(\frac{\mathcal{F}_{N+1}}{\mathcal{F}_{N}}\right)
$$

Now we define an inverse squared length scale:

$$
\lambda_{0}^{-2}=\lambda_{f}^{-2}+R^{-2}
$$

In all the future calculations the domain of integration is assumed to be $S^{2}$.

\section{Ladder estimates}

Let us start with $H_{0}$. By definition,

$$
H_{0}=\int \varphi^{2} d S \Rightarrow \frac{1}{2} \dot{H}_{0}=\int \varphi_{t} \varphi d S
$$

After substitution of $\varphi_{t}$ from equation (6) we obtain

$$
\frac{1}{2} \dot{H}_{0}=\nu \int \varphi \Delta \varphi d S-\int \varphi J\left(\Delta^{-1} \varphi, \varphi+l\right) d S+\int\left(\vec{n} \cdot \operatorname{rot}_{n} f\right) \varphi d S
$$

The last term in (10) can be estimated with the help of the Cauchy-Schwarz inequality as follows,

$$
\int\left(\vec{n} \cdot \operatorname{rot}_{n} f\right) \varphi d S \leq\left(\int\left(\vec{n} \cdot \operatorname{rot}_{n} f\right)^{2} d S\right)^{1 / 2}\left(\int \varphi^{2} d S\right)^{1 / 2}=\mathcal{F}_{0}^{1 / 2} H_{0}^{1 / 2} .
$$


Now applying equality (3) to the Laplacian term in (10) one obtains:

$$
\nu \int \varphi \Delta \varphi d S=\nu \int \varphi \operatorname{div} \nabla \varphi d S=-\nu \int(\nabla \varphi)^{2} d S=-\nu H_{1}
$$

The last term we need to estimate is the nonlinear term in (10). Using the properties of $J(a, b)$ and recalling that $\Delta^{-1} \varphi=\psi$ gives us the following result:

$$
-\int \varphi J\left(\Delta^{-1} \varphi, \varphi+l\right) d S=-\int \varphi J(\psi, \varphi) d S-\int J(\psi, l) \varphi d S=0
$$

Adding all the above calculations we can write the inequality for the "bottom" rung of the ladder as

$$
\frac{1}{2} \dot{H}_{0}=-\nu H_{1}+\mathcal{F}_{0}^{1 / 2} H_{0}^{1 / 2}
$$

This form of ladder is inconvenient to leave because of the way the forcing term is expressed in here. Later on, we will want to divide through by $H_{0}$ and the square root of forcing will cause complications. For this reason we introduce $F_{0}$ and $(11)$ can be rewritten as:

$$
\frac{1}{2} \dot{F}_{0} \leq-\nu F_{1}+\nu \lambda_{0}^{-2} F_{0}
$$

As before, consider the next quantity $H_{1}$. Substituting $\varphi_{t}$ from the equation (6) one gets:

$$
\begin{aligned}
\frac{1}{2} \dot{H}_{1}=\int \nabla \varphi \nabla \varphi_{t} d S & =\nu \int \nabla \Delta \varphi \nabla \varphi d S-\int \nabla J\left(\Delta^{-1} \varphi, \varphi+l\right) \nabla \varphi d S \\
& +\int \nabla\left(\vec{n} \cdot \operatorname{rot}_{n} f\right) \nabla \varphi d S .
\end{aligned}
$$

The Laplacian term in (13) can be treated as follows,

$$
\nu \int \nabla \Delta \varphi \nabla \varphi d S=-\nu \int \Delta \varphi \operatorname{div} \nabla \varphi d S=-\nu \int(\Delta \varphi)^{2} d S=-\nu H_{2}
$$

The second term in (13) is:

$$
-\int \nabla J\left(\Delta^{-1} \varphi, \varphi+l\right) \nabla \varphi d S=\int J\left(\Delta^{-1} \varphi, \varphi+l\right) \operatorname{div} \nabla \varphi d S
$$




$$
=\int J\left(\Delta^{-1} \varphi, \varphi+l\right) \Delta \varphi d S=\int J\left(\Delta^{-1} \varphi, \varphi\right) \Delta \varphi d S+\int J\left(\Delta^{-1} \varphi, l\right) \Delta \varphi d S .
$$

By definition, $J(a, b)=\vec{n} \times \nabla a \cdot \nabla b$. This gives,

$$
\begin{aligned}
\int J\left(\Delta^{-1} \varphi, \varphi\right) \Delta \varphi d S & =\int(\vec{n} \times \nabla \psi \cdot \nabla \varphi) \Delta \varphi d S \\
& \leq \int|\nabla \psi\|\nabla \varphi\| \Delta \varphi| d S \leq\|\nabla \psi\|_{\infty} \int|\nabla \varphi \| \Delta \varphi| d S
\end{aligned}
$$

After application of the Cauchy-Schwartz and then Young's inequalities, we obtain

$$
\begin{aligned}
\int J\left(\Delta^{-1} \varphi, \varphi\right) \Delta \varphi d S & \leq \frac{\nu}{2} \int(\Delta \varphi)^{2} d S+\frac{2}{\nu}\|\nabla \psi\|_{\infty}^{2} \int(\nabla \varphi)^{2} d S \\
& =\frac{\nu}{2} H_{2}+\frac{2}{\nu}\|\nabla \psi\|_{\infty}^{2} H_{1} .
\end{aligned}
$$

With the help of the interpolation inequality [6]

$$
\|f\|_{\infty} \leq \frac{1}{2}\|f\|_{2}^{1 / 2}\|\Delta f\|_{2}^{1 / 2}
$$

and then Poincaré's inequality, $\|\nabla \psi\|_{\infty}$ in (14) becomes

$$
\|\nabla \psi\|_{\infty}^{2} \leq \frac{1}{4}\|\nabla \psi\|_{2}\left\|\nabla^{3} \psi\right\|_{2} \leq \frac{1}{4 \sqrt{2}}\|\Delta \psi\|_{2}\left\|\nabla^{3} \psi\right\|_{2}
$$

Substituting the last estimate into (14) gives

$$
\frac{\nu}{2} H_{2}+\frac{1}{2 \sqrt{2} \nu}\|\Delta \psi\|_{2}\left\|\nabla^{3} \psi\right\|_{2} H_{1}=\frac{\nu}{2} H_{2}+\frac{1}{2 \sqrt{2} \nu} H_{0}^{1 / 2} H_{1}^{1 / 2} H_{1}=\frac{\nu}{2} H_{2}+\frac{1}{2 \sqrt{2} \nu} H_{0}^{1 / 2} H_{1}^{3 / 2}
$$

Hence,

$$
\int J\left(\Delta^{-1} \varphi, \varphi\right) \Delta \varphi d S \leq \frac{\nu}{2} H_{2}+\frac{1}{2 \sqrt{2} \nu} H_{0}^{1 / 2} H_{1}^{3 / 2} .
$$

Moreover, it can be established that

$$
\int J(\psi, l) \Delta \varphi d S=\int J(\psi, l) \Delta^{2} \psi d S=0
$$

In fact we have

$$
\Delta^{2} \partial_{\theta} \psi=\partial_{\theta} \Delta^{2} \psi \text {, and } J(\psi, l)=2 \omega \partial_{\theta} \psi
$$


After integration by parts with respect to $\theta$, we obtain

$$
\left(\partial_{\theta} \psi, \Delta^{2} \psi\right)=-\left(\psi, \partial_{\theta} \Delta^{2} \psi\right)=-\left(\psi, \Delta^{2} \partial_{\theta} \psi\right)
$$

On the other hand, using the fact that the Laplacian is a self-adjoint operator, gives

$$
\left(\partial_{\theta} \psi, \Delta^{2} \psi\right)=\left(\Delta^{2} \partial_{\theta} \psi, \psi\right)=\left(\psi, \Delta^{2} \partial_{\theta} \psi\right)
$$

This proves the orthogonality relation (15) and concludes the computation of the second term in (13). Finally, consider the last term in (13)

$$
\int \nabla\left(\vec{n} \cdot \operatorname{rot}_{n} f\right) \nabla \varphi d S \leq\left(\int\left(\nabla\left(\vec{n} \cdot \operatorname{rot}_{n} f\right)\right)^{2} d S\right)^{1 / 2}\left(\int(\nabla \varphi)^{2}\right)^{1 / 2}=\mathcal{F}_{1}^{1 / 2} H_{1}^{1 / 2}
$$

By virtue of all the above calculations one has

$$
\frac{1}{2} \dot{H}_{1} \leq-\frac{\nu}{2} H_{2}+\frac{1}{2 \sqrt{2} \nu} H_{0}^{1 / 2} H_{1}^{3 / 2}+\mathcal{F}_{1}^{1 / 2} H_{1}^{1 / 2}
$$

or, in terms of $F_{N}$, this ladder inequality becomes

$$
\frac{1}{2} \dot{F}_{1} \leq-\frac{\nu}{2} F_{2}+\frac{1}{2 \sqrt{2} \nu} F_{0}^{1 / 2} F_{1}^{3 / 2}+\lambda_{0}^{-2} \nu F_{1}
$$

\section{Length scales}

In [8] and [9] the length scales for the Navier-Stokes equations on flat domains and for the complex Ginzburg-Landau equation were derived using the Fourier expansion of the solution in order to obtain the number of relevant modes $\kappa_{N, r}$.

Definition 1 [10]. Define the "wavenumbers" as the following ratio

$$
\kappa_{N, r}(t)=\left(\frac{F_{N}}{F_{r}}\right)^{\frac{1}{2(N-r)}}, \text { with } N=1,2, \text { and } r=0,1 \text {. }
$$


If we divide the ladder inequality for $F_{0}\left(F_{1}\right)$ through by $F_{0}\left(F_{1}\right)$, then the square of $\kappa_{1,0}\left(\kappa_{2,1}\right)$ appears in the first term on the right hand side of inequalities (12) and (17), respectively.

Definition 2 [10]. We will now define the length scales as the time average of the inverse squared wavenumbers

$$
\ell_{N, r}^{-2} \equiv\left\langle\kappa_{N, r}^{2}\right\rangle=\left\langle\left(\frac{F_{N}}{F_{r}}\right)^{\frac{1}{(N-r)}}\right\rangle \text {, with } N=1,2 \text {, and } r=0,1 \text {, }
$$

where the time average $\langle g\rangle$ is defined as

$$
\langle g\rangle=\lim _{t \rightarrow \infty} \frac{1}{t} \int_{0}^{t} g(\tau) d \tau
$$

Recasting the estimate (11) in the following form

$$
\frac{d}{d t}\left(\frac{1}{2}\|\varphi\|_{2}^{2}\right)=-\nu\|\nabla \varphi\|_{2}^{2}+\|\varphi\|_{2}\left\|\vec{n} \cdot \operatorname{rot}_{n} f\right\|_{2}
$$

and using the Poincaré inequality we obtain

$$
\frac{d}{d t}\left(\frac{1}{2}\|\varphi\|_{2}^{2}\right) \leq-\frac{\nu}{4}\|\nabla \varphi\|_{2}^{2}+\frac{1}{\nu}\left\|\vec{n} \cdot \operatorname{rot}_{n} f\right\|_{2}^{2}
$$

The application of Gronwall's lemma to the last expression gives

$$
\varlimsup_{t \rightarrow \infty}\|\varphi\|_{2}^{2} \leq \frac{4}{\nu^{2}}\left\|\vec{n} \cdot \operatorname{rot}_{n} f\right\|_{2}^{2}
$$

The expression (18) is only a part of what we need in order to obtain the estimates for the bottom rung of the ladder, i.e. for $F_{0}$. From $(7)$ one recalls that

$$
F_{0}=\|\varphi\|_{2}^{2}+\nu^{-2} R^{4}\left\|\vec{n} \cdot \operatorname{rot}_{n} f\right\|_{2}^{2}
$$

Together with (18) this gives

$$
\varlimsup_{t \rightarrow \infty} F_{0} \leq \frac{4}{\nu^{2}}\left\|\vec{n} \cdot \operatorname{rot}_{n} f\right\|_{2}^{2}+\nu^{-2} R^{4}\left\|\vec{n} \cdot \operatorname{rot}_{n} f\right\|_{2}^{2}
$$


Returning now to the ladder estimate for $F_{0}$ in form of (12), we may time average this ladder to obtain $\left\langle F_{1}\right\rangle$ or divide by $F_{0}$ and then time average to find $\left\langle\kappa_{1,0}^{2}\right\rangle$. For both cases the results are the following

$$
\left\langle F_{1}\right\rangle \leq \frac{\lambda_{0}^{-2}}{\nu^{2}}\left\|\vec{n} \cdot \operatorname{rot}_{n} f\right\|_{2}^{2}\left(4+R^{4}\right)
$$

and

$$
\ell_{1,0}^{-2} \equiv\left\langle\kappa_{1,0}^{2}\right\rangle \leq\left\langle\frac{F_{1}}{F_{0}}\right\rangle \leq \lambda_{0}^{-2}
$$

It is worth noting that the estimate (20) includes $\lambda_{0}^{-2}$ and, therefore, takes account of spectral information of the forcing.

Using the same technique as before and employing now (17) we again are able to find the time average estimate for $\left\langle\kappa_{1,0}^{2}\right\rangle$ as follows,

$$
\ell_{2,1}^{-2} \equiv\left\langle\kappa_{2,1}^{2}\right\rangle \leq\left\langle\frac{F_{2}}{F_{1}}\right\rangle \leq \frac{1}{\sqrt{2} \nu^{4}}\left(4+R^{4}\right) \lambda_{0}^{-1}\left\|\vec{n} \cdot \operatorname{rot}_{n} f\right\|_{2}^{2}+2 \lambda_{0}^{-2}
$$

\section{Conclusions}

When applying the ladder method to dissipative partial differential equations on a non-planar domain, in particular on the rotating sphere, one has to deal with different boundary conditions than the periodic ones or no boundary conditions at all.This introduces considerable difficulties. In this letter we avoid these difficulties by replacing the original Navier-Stokes equations on the 2-D rotating sphere by the vorticity equation. Using the vorticity equation we applied the ladder method and found bounds for the smallest length scales in the solutions which arise naturally from the ladder structure. Besides, we have also obtained the estimates for the $L^{2}$-norm of $H_{0}$ and $H_{1}$, and it is worth noting that all estimates do not contain any unknown constants. Due to the 
restricted applicability of interpolation inequalities on the sphere, the analysis for the $L^{2}$-norm of higher derivatives of the solutions is much more involved. This is currently work in progress.

\section{References}

[1] S. Sukoriansky, B. Galperin and N. Dikovskaya, Phys. Rev. Lett. 89 (2002) 124501

[2] A.A. Ilyin, Differential Integral Equations 6 (1993) 183.

[3] A.A. Ilyin, Nonlinearity 7 (1994) 31.

[4] C. Cao, M.A. Rammaha and E.S. Titi, Z. angew. Math. Phys. 50 (1999) 341.

[5] A.N. Filatov, A.A. Ilyin, Soviet Math. Dokl. 38 (1989) 18.

[6] A.A. Ilyin, J. London Math. Soc. 2 (1999) 263.

[7] J.-M. Ghidaglia, SIAM J. Math. Anal. 17 (1986) 1139.

[8] M.V. Bartuccelli, J.D. Gibbon and M. Oliver, Physica D 44 (1996) 267.

[9] M.V. Bartuccelli, C.R. Doering, J.D. Gibbon and S.J.A. Malham, Nonlinearity $6(1993) 549$.

[10] C.R. Doering, J.D. Gibbon, Applied analysis of the Navier-Stokes equations (CUP, Cambridge, 1995). 